Article

\title{
Association of Circulating Tumor Cells with Inflammatory and Biomarkers in the Blood of Patients with Metastatic Castration-Resistant Prostate Cancer
}

\author{
Gerit Theil *, Carlotta Lindner, Joanna Bialek and Paolo Fornara \\ Medical Faculty of Martin Luther University Halle-Wittenberg, University Clinic and Outpatient Clinic for \\ Urology, 06120 Halle (Saale), Germany; Carlotta.lindner@uk-halle.de (C.L.); joanna.bialek@uk-halle.de (J.B.); \\ paolo.fornara@uk-halle.de (P.F.) \\ * Correspondence: Gerit.Theil@medizin.uni-halle.de
}

check for updates

Citation: Theil, G.; Lindner, C.; Bialek, J.; Fornara, P. Association of Circulating Tumor Cells with Inflammatory and Biomarkers in the Blood of Patients with Metastatic Castration-Resistant Prostate Cancer. Life 2021, 11, 664. https://doi.org/ $10.3390 /$ life11070664

Academic Editors: Ana Faustino and Paula A. Oliveira

Received: 25 May 2021

Accepted: 4 July 2021

Published: 6 July 2021

Publisher's Note: MDPI stays neutral with regard to jurisdictional claims in published maps and institutional affiliations.

Copyright: (c) 2021 by the authors. Licensee MDPI, Basel, Switzerland. This article is an open access article distributed under the terms and conditions of the Creative Commons Attribution (CC BY) license (https:// creativecommons.org/licenses/by/ $4.0 /)$.

\begin{abstract}
The identification of specific biomarkers that recognize the functional drivers of heterogeneity in prostate cancer (PCa) and personalized treatment remain challenging in systemic medicine. Liquid biopsy allows for the detection and analysis of personalized predictive biomarkers in single blood samples and specifies the current stage of cancer. The aim of our preliminary study was to investigate the association between an elevated circulating tumor cell (CTC) count and the levels of inflammatory factors (IL-6 and IL-8) and biomarkers (DKK-1, PSA, sHER2, and CD44) in patients with metastasized castration-resistant PCa (mCPRC) under chemotherapy and those with localized PCa. Such an association could be used as a component of cancer progression monitoring. We compared the sensitivity and specificity of two CTC isolation platforms. Twenty-eight patients (12 mCRPC and 16 localized PCa patients) were enrolled. Over the study period, the CTC detection rates were $84 \%$ with CellCollector ${ }^{\circledR}$ and $73.5 \%$ with CellSearch ${ }^{\circledR}$ System in mCPRC patients. The CTC counts determined by the CellSearch ${ }^{\circledR}$ System (CTC_CS) were correlated significantly with the DKK-1, sHER-2, and PSA concentrations in mCRPC patients. The CTC counts captured by CellCollector ${ }^{\circledR}$ demonstrated no significant association with the concentrations of the tested blood-based biomarkers. The CTC_CS count (AUC $=0.9$ (95\% CI: 0.72-1.0)) and the PSA level (AUC $=0.95$ (95\% CI: 0.83-1.0)) presented approximately the same sensitivity and specificity for the overall survival of mCRPC patients. For better personalized characterization, further research on CTC phenotyping and their interactions with tumor-associated blood-released factors is needed.
\end{abstract}

Keywords: biomarker; circulating tumor cells; prostate cancer

\section{Introduction}

Prostate cancer (PCa) is the fifth leading cause of cancer-related death worldwide [1]. The incidence increases with each decade of age, and thus, 59\% of men over 79 years of age have PCa [2]. In an aging population, more PCa would be diagnosed. Furthermore, in men aged 75 years and older, the incidence of regional- and distant-stage disease increased from 2013 to 2016 [3]. For $28 \%$ of patients with distant metastasis, the estimated survival rate is approximately 5 years [4]. The majority of these patients have multifocal metastatic sites, such as bone and lymph nodes (particularly vertebrae and pelvis) [5]. Moreover, oligometastatic PCa has distinct biological states and harbors different mutations, which result in heterogeneous phenotypes. Metastatic progression requires certain characteristics of cancer cells, such as plasticity, motility, and colonization, as well as systemic physiological conditions, such as inflammation, which are drivers of metastasis and therapeutic resistance in PCa [6]. Drug resistance is a dynamic process in tumor cells, which includes molecular events such as genome modification and the regulation of diverse transcriptional states. Additionally, cancer cells undergo phenotype acquisition in the process of cellular rewiring [7]. In the last decade, innovations in treatments and combination therapeutic 
strategies have been developed and have contributed to the therapeutic armamentarium, improving the outcomes from metastatic PCa $[8,9]$.

Nevertheless, the determination of the optimal personalized drug sequence to minimize possible therapeutic resistance remains a challenge [10]. Therefore, personalized biomarkers of these characteristics are needed to determine treatment responses and facilitate decisions on the selection of agents.

Classic clinical factors, such as the blood levels of prostate-specific antigen (PSA), and pathological factors, such as Gleason grading and tumor, node, and metastasis (TNM) staging, are well-known prognostic markers in PCa [11]. However, these methods are often insufficient for accurate risk stratification, and do not adequately describe the metastatic process. One possibility is liquid biopsy, which includes (among others) circulating tumor cells (CTCs). CTCs detach from primary or metastatic tumors to enter the bloodstream, and a small CTC population has the ability to metastasize to multiple organs [12]. They provide characteristics of the current stage of the tumor or potential metastasis and allow for the real-time monitoring of therapeutic responses. CTCs' interplay with blood components is important for their survival and metastatic characteristics $[13,14]$. They may interact with neutrophils, platelets, leukocytes, monocytes, and macrophages in the circulation, which protect the CTCs from rapid clearance by natural killer cells and the physical shear stress of blood flow. These interactions promote the survival and extravasation of CTCs at distant sites [13].

The cytokines interleukin 8 (IL-8) and IL-6 are associated with inflammation contributing to PCa and progression to treatment resistance. IL-8 is secreted by monocytes, neutrophils, and endothelial cells. Its signaling in PCa cells is involved in regulating the transcriptional activity of the androgen receptor (AR), and substantiates the transition to an androgen independent proliferation of prostate cancer cells [15]. Furthermore, IL-8 overexpression by tumor cells is often induced in response to chemotherapeutic treatment and may be important in the tumor microenvironment [16,17].

IL-6 stimulates proliferation, promotes angiogenesis, and inhibits apoptosis of $\mathrm{PCa}$ cells and other tumor cells. These activities are due to the interaction of IL-6 with multiple signaling pathways, such as the Janus tyrosine family kinase (JAK)-signal transducer and activator of transcription (STAT) pathway and the extracellular signal-regulated kinase 1 and 2 (ERK1/2)-mitogen activated protein kinase (MAPK) pathway [18]. Additionally, IL-6 has been identified as a nonsteroidal compound of AR activation (N-terminus of AR), which is different from ligand activation $[19,20]$. IL-6 is also known to induce human epidermal growth factor receptor-2 (HER2) signaling through the MAPK pathways [21]. HER2 belongs to the epidermal growth receptor family, which regulates processes such as cell differentiation, migration, and survival. The activation of HER2 results in ligandindependence over homodimerization, heterodimerization with other receptors of the HER family, or proteolytic cleavage of the extracellular domain (sHER2 ECD) [22]. HER2 signaling promotes AR signaling through androgen ligand-independent mechanisms and supports the development of castration-resistant PCa (CRPC) [23,24]. Ma et al. [25] demonstrated that CD44 interacted with HER2 promotes DNA damage repair and radioresistance. Moreover, CD44 expression in cancer cells promotes bone metastases by enhancing tumorigenicity, cell migration, and progression [26,27].

CRPC patients mostly have bone metastasis, which results in skeletal-related events such as pathological fractures. Osteoblast function is dependent on Wnt signaling, controlled by the Wnt inhibitors sclerostin and Dickkopf1 (DKK-1) [28]. Furthermore, DKK-1 expression in tumor cells activated $\mathrm{Wnt} / \beta$-catenin signaling and demonstrated an interaction with AR signaling [29].

The aim of our preliminary study was to investigate the association of an elevated CTC count with inflammatory molecules (IL-6 and IL-8) and biomarkers (DKK-1, sHER2, and CD44) in patients with metastasized CPRC (mCPRC) under chemotherapy and localized PCa (PCa-1). Such an association could be used as a component of cancer progression monitoring. 


\section{Materials and Methods}

\subsection{Patient Cohorts}

This is a retrospective analysis of a subpopulation of a prospectively planned clinical trial in the University Clinic and Outpatient Clinic for Urology, Medical Faculty of Martin Luther University Halle-Wittenberg [30]. All of the patients provided written informed consent and were enrolled in the study. This included blood sampling ( $4.5 \mathrm{~mL}$ serum) for future research. The protocol was approved by the medical faculty ethics committee of Martin Luther University Halle-Wittenberg (number of ethical approval: FSMW EPCAMProstata-M00, 2012-65). The men enrolled in the first group were patients with histologically confirmed prostate adenocarcinoma with progressive disease, despite castration levels of serum testosterone $(<50 \mathrm{ng} / \mathrm{dL})$. Only two of the patients achieved the castration-resistant stage in the second month of the study. All 12 patients were examined every month for 6 months, followed by visits in the 8th and 12th months, for a total of eight visits. CTC evaluation with CellCollector ${ }^{\circledR}$ and the CellSearch ${ }^{\circledR}$ System and blood sampling for additional biomarker analysis were taken before starting the chemotherapy or the bone-targeted therapy. The second group included patients with confirmed prostate adenocarcinoma, who had opted for radical prostatectomy (RP) in the observation period and were assessed three times within 12 months. The first visit was before the prostate removal. The next visits were 6 and 12 months after surgery.

\subsection{Sample Collection}

Additionally, $9 \mathrm{~mL}$ of blood serum was collected for the determination of the levels of PSA, C-reactive protein (CRP), and testosterone and for Luminex analysis. Samples were collected at each visit. The serum was processed within one hour after collection through centrifugation at $1300 \times g$ for $10 \mathrm{~min}$. The samples were stored at $-80^{\circ} \mathrm{C}$.

\subsection{CTC Isolation}

We used two different methods for CTC isolation, the CellSearch ${ }^{\circledR}$ System (Silicon Biosystem, Menarini, Florence, Italy) and CellCollector ${ }^{\circledR}$ (GILUPI GmbH, Potsdam, Germany), at matched times. Both systems used an epithelial cell adhesion molecule (EpCAM) antibody to capture the CTCs, as previously described [30,31].

CellCollector ${ }^{\circledR}$, a medical wire, was carefully inserted into the patient's cubital vein via a $20 \mathrm{G}$ peripheral venous catheter until the tip of the wire $(2 \mathrm{~cm})$ was in the bloodstream of the vein. After $30 \mathrm{~min}$, the wire was pulled out of the vein. In the first step, the captured cells were fixed with $100 \%$ acetone for $10 \mathrm{~min}$ at room temperature, blocked with $3 \%$ bovine serum albumin/PBS for $30 \mathrm{~min}$, and then prepared for characterization.

For the CellSearch ${ }^{\circledR}$ System analysis, $7.5 \mathrm{~mL}$ of blood was collected in CellSave ${ }^{\circledR}$ Preservative Tubes. These samples remained stable for $96 \mathrm{~h}$ at room temperature and were sent overnight to the Department of Tumor Biology, University Medical Center Hamburg-Eppendorf, Hamburg, Germany.

\subsection{CTC Characterization}

The matched pair analysis requires the same identification criteria as that of the CTCs. The captured cells were stained with fluorescein isothiocyanate (FITC)-labeled antibodies against cytokeratin 8, 18, and 19 (eBioscience, Abcam) for the detection of epidermal cancer cells in the blood. CD45 staining (anti-CD45-A647, Exbio) was performed to exclude leucocytes. Additionally, the cells' nuclei were stained with Hoechst 33342. Cells were defined as CTCs when they met the following cytology-based FDA definition: (i) size $\geq 4 \mu \mathrm{m}$, (ii) visible cytoplasm, (iii) high nuclear/cytoplasm ratio, and (iv) positive fluorescent staining, as described above [32,33].

The images were digitally processed with ImageJ software by altering the contrast and brightness in accordance with Nature Publishing Guidelines [34]. 


\subsection{Detection of Circulating Biomarkers}

The serum levels of sHER2, IL-8, IL-6, DKK-1, and CD44 were simultaneously determined by a custom-made configuration of the Luminex Screening Human Magnetic Assay (R\&D Systems). The assays were conducted following the manufacturer's instructions and were performed on a Luminex $100^{\mathrm{TM}}$ Qiagen $\mathrm{GmbH}$ system (Hilden, Germany). All of the serum samples required a two-fold dilution in calibration diluent. For the analysis, we used a 96-well flat bottom microplate. The measurements for each sample were performed in duplicate, and the average of the two measurements was used. Limits of quantification were determined using the lowest or highest standard point and a percent $\mathrm{CV}(\% \mathrm{CV}=$ $100 \times$ standard deviation/average) of less than $20 \%$. PSA and CRP were determined with Immulite 100 (Siemens Healthcare Diagnostics GmbH, Eschborn, Germany), according to the manufacturer's instructions.

\subsection{Statistical Analysis}

All of the determined blood-based biomarkers or metabolites were normalized. The values obtained at the first visits were defined as $100 \%$. The relative secretion values are shown in box plots with medians and interquartile ranges (IQRs). Whiskers represent the minimum and maximum values. Furthermore, all of the data were tested for normal distribution using the Shapiro-Wilk test, and the parameters are presented as the median \pm range.

Finally, for the identification of possible correlations between the different markers for the different study groups, Spearman's rank correlation coefficient $\left(\mathrm{r}_{\mathrm{s}}\right)$ was determined and is represented in a heatmap. The reported p-values are two-sided, and $\leq 0.05$ was considered significant. The accuracy of the selected biomarker levels was evaluated by receiver operating characteristic (ROC) analysis. For this analysis, we used no cut-offs, but the median was six visits performed for 24 months survival. The optimal cut-off for the Kaplan-Meier analysis of PSA based on the ROC curve was calculated by the largest value of the formula, sensitivity + specificity -1 , from the median PSA level for every mCRPC patient (likelihood ratio). The mean CTC count was determined based on the CTC counts of visits 1-8 (V1-V8). Kaplan-Meier analysis was used to analyze the overall survival (OS) depending on the mean CTC count. The survival estimates in different groups were compared using the log-rank (Mantel-Cox) test. All of the statistical analyses were performed using GraphPad Prism software versions 7 and 9.

\section{Results}

\subsection{Study Design and Patient Data}

A total of 28 patients (12 mCRPC patients and16 PCa-l patients) were enrolled in the analysis. All of the study-related applications were identical in the groups. Age $(p=0.09)$ and body mass index $(p=0.18)$ were not significantly different between the groups (Mann-Whitney test). The median age was 68.5 years in the MCRPC patients and 63 years in the PCa-1 patients. The median BMI was 27.5 in the mCRPC patients and 29.7 in the PCa-l patients. The Gleason score was significantly different $(p<0.0001)$ between the PCa-1 and mCRPC groups (Mann-Whitney test). Ten patients (83.3\%) received docetaxel in combination with prednisone as the first-line treatment for $\mathrm{mCRPC}$, and three $(25 \%)$ received cabazitaxel (one patient switched in the study period from docetaxtel to cabazitaxel) in response to resistance to docetaxel. The PCa-l patients were treated after the first visit with laparoscopic RP (82.3\%) or high-intensity focused ultrasound (HIFU) $(11.76 \%)$. The other baseline characteristics are summarized in Table 1. 
Table 1. Study population characteristic and demographics.

\begin{tabular}{|c|c|c|}
\hline Characteristics & mCRPC & PCa-1 \\
\hline Patient $(n)$ & 12 & 16 \\
\hline Median (range), years & $69(53-72)$ & $63(56-75)$ \\
\hline Median (range) BMI & $27.5(20.8-39)$ & $29.7(22.5-34.5)$ \\
\hline Median PSA (range), $\mathrm{ng} / \mathrm{mL}$ at baseline & $25.6(35-1200)$ & $8.2(0.64-38.8)$ \\
\hline Median PSA (range), $\mathrm{ng} / \mathrm{mL}$ at the last visit & $44.95(0.04-903)$ & $0,04(0.04-0.06)$ \\
\hline $\begin{array}{c}\text { Median CRP (range), } \mathrm{mg} / \mathrm{mL} \\
\text { at baseline }\end{array}$ & $7.3(1.8-94.8)$ & $2.3(1-26.2)$ \\
\hline $\begin{array}{l}\text { Median HB (range), nmol/L } \\
\text { at baseline }\end{array}$ & $7(6.3-9.5)$ & $9.4(7.9-10.5)$ \\
\hline \multicolumn{3}{|l|}{ Gleason sum, n (\%) } \\
\hline$\leq 7$ & $2(16.67)$ & $11(64.7)$ \\
\hline$>7$ & $10(83.33)$ & $6(35.3)$ \\
\hline \multicolumn{3}{|l|}{ Sites of metastasis, $n(\%)$} \\
\hline Bone & $12(100)$ & \\
\hline Visceral & $4(33.3)$ & \\
\hline Nodal & $10(83.3)$ & \\
\hline \multicolumn{3}{|l|}{ Prior treatments, $\mathrm{n}(\%)$} \\
\hline TURP & $5(41.7)$ & \\
\hline Androgen treatment & $12(100)$ & \\
\hline Radiation & $9(75)$ & \\
\hline \multicolumn{3}{|l|}{ Treatments between baseline and study end, $n(\%)$} \\
\hline \multicolumn{3}{|l|}{ TURP } \\
\hline Surgery (RP) & & $14(82.3)$ \\
\hline HIFU & & $2(11.8)$ \\
\hline Radiation & $10(83.3)$ & \\
\hline Bone-targeted therapy & $12(100)$ & \\
\hline \multicolumn{3}{|l|}{ Chemotherapy } \\
\hline Docetaxel & $10(83.3)$ & \\
\hline Cabazitaxel & $3 *(25)$ & \\
\hline
\end{tabular}

RP-radical prostatectomy; HB-hemoglobin; PSA—-prostate-specific antigen; TURP—transurethral resection of the prostate; HIFU-highintensity focused ultrasound; BMI—body mass index; CRP—C-reactive protein. 3 * one CRPC patient received docetaxel and switched to cabazitaxel during the study period.

\subsection{Assessment of Different Serum and Blood Biomarkers}

We isolated CTCs with two different EpCAM-based systems from the mCPRC $(n=12)$ and PCa-l $(n=16)$ patients. Over the study period, the CTC detection rates were $84 \%$ with CellCollector ${ }^{\circledR}$ (CTC_CC) and 73.5\% with the CellSearch ${ }^{\circledR}$ system (CTC_CS) in the mCPRC patients. Furthermore, the CTC-median in the mCRPC patients did not differ significantly $(p=0.29)$ between the two isolation platforms. A median of 4 CTCs (range 0-820) was captured by CellCollector ${ }^{\circledR}$, and 8.5 CTCs (range 0-1428) by the CellSearch ${ }^{\circledR}$ system (Figure 1a). The baseline CTC count was zero in one mCPRC patient with CellCollector ${ }^{\circledR}$ and in three patients with the CellSearch ${ }^{\circledR}$ system. At the first visit, seven patients $(58.8 \%)$ had $<5$ CTCs and three $(25 \%)$ had $\geq 5$ CTCs, as determined with CellCollector ${ }^{\circledR}$. When the CellSearch ${ }^{\circledR}$ system was used, one (8.3\%) patient had $<5$ CTCs and nine $(75 \%)$ patients had $\geq 5$ CTCs.

The PCa-l group had a median of 0 CTCs detected with both platforms at the first visit; 0-5 CTCs were achieved with CellCollector ${ }^{\circledR}$ and 0-1 CTCs were achieved with the CellSearch ${ }^{\circledR}$ system. In addition, in the cured patients, 0 CTCs were detected using the

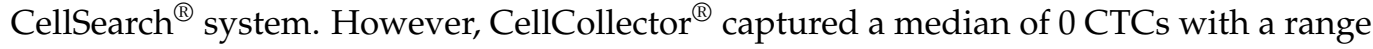
of $0-9$ at visits 6 and 12 months after RP (Figure 1b). 

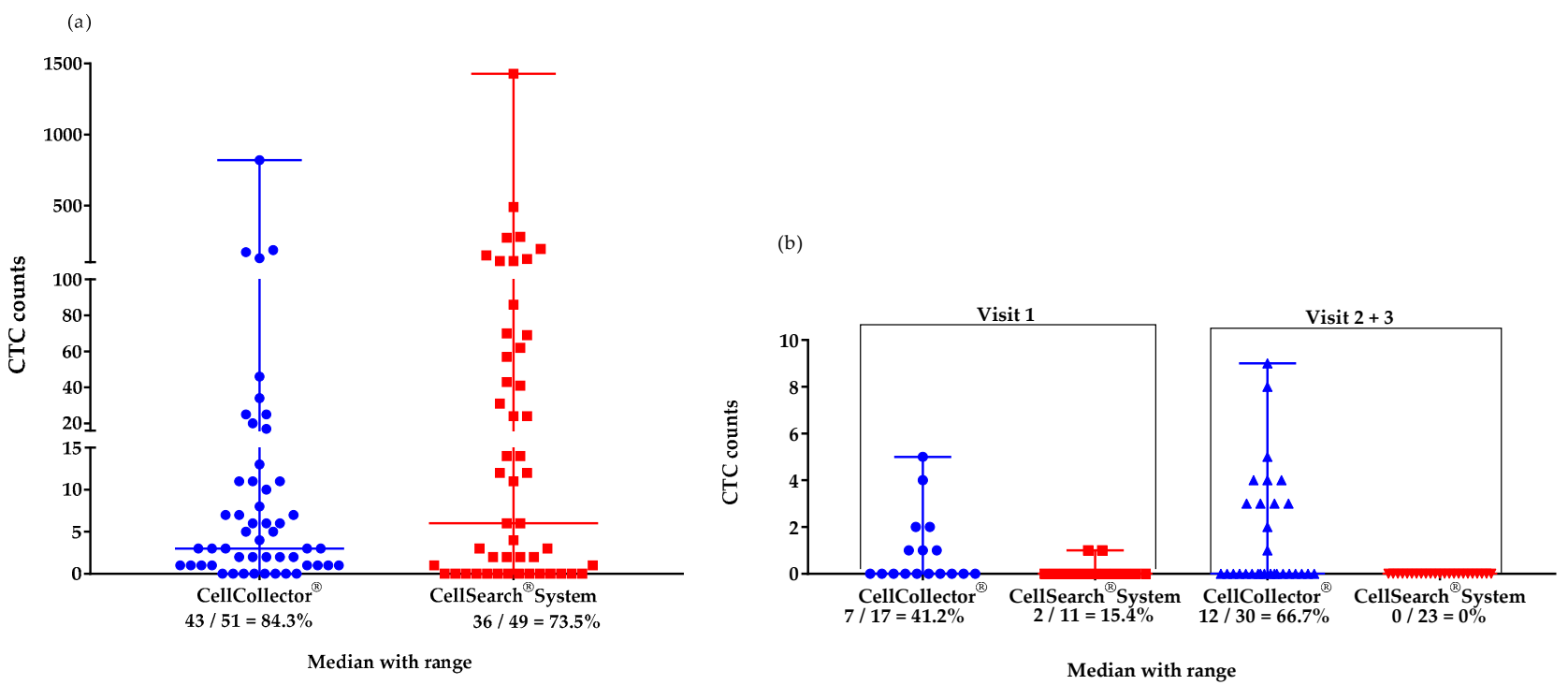

Figure 1. Median (range) values of CTCs isolated with the CellCollector ${ }^{\circledR}$ and CellSearch ${ }^{\circledR}$ Systems. (a) mCRPC patients $(n=12)$ in the study period of 12 months: CellCollector ${ }^{\circledR}, 4$ CTCs $(0-820)$, and CellSearch ${ }^{\circledR}$ System, 8.5 CTCs $(0-1428)$; (b) PCa-l patients $(n=16)$ at visit 1 (before surgical removal): CellCollector ${ }^{\circledR}, 0$ CTCs $(0-5)$, and CellSearch ${ }^{\circledR}$ System, 0 CTCs (0-1) and at visits 2 and 3 (6 and 12 months after removal of prostate, respectively): CellCollector ${ }^{\circledR}, 0$ CTCs (0-9), and CellSearch ${ }^{\circledR}$ System, 0 CTCs (0).

Biomarkers were measured until visit 6 (sixth month) in the study period. Unfortunately, data from visits 7 and 8 could not be included in the analysis because of an insufficient sample size. The serum levels of sHER2, IL-8, IL-6, Dkk-1, and CD44 did not show significant differences between the PCa-1 and mCRPC patients (Table 2). The median levels of DKK-1 (4625 pg/mL), IL-6 (11.7 pg/mL), and IL-8 $(20 \mathrm{pg} / \mathrm{mL})$ in the mCRPC patients were higher than those in the PCa-1 patients $(3939 \mathrm{pg} / \mathrm{mL}, 5.6 \mathrm{pg} / \mathrm{mL}$, and $10.8 \mathrm{pg} / \mathrm{mL}$, respectively). Interestingly, the CD44 level in the mCRPC patients was the lowest in the study population. Moreover, the sHER level demonstrated a decreased concentration over six months in the mCRPC group. The median secretion levels were $3.3 \mathrm{ng} / \mathrm{mL}$ in the PCa-l group at visit 1 and $3.5 \mathrm{ng} / \mathrm{mL}$ at visits 2 and 3 . The mCRPC patients had a median concentration of $3.3 \mathrm{ng} / \mathrm{mL}$, which was approximately equal to the concentrations in the localized cancer stage groups. Interestingly, the sHER concentration had the widest range of $0.3-16.64 \mathrm{ng} / \mathrm{mL}$ in the mCRPC group. Significant differences were found for the PSA level $(p<0.001)$, CRP level $(p=0.03)$, and CTC count $(p<0.001)$ between the groups (Table 2$)$.

We investigated the serial secretion of the biomarkers in the treatment follow-up at 6 months in the mCRPC patients (Figure 2). The first values were defined as $100 \%$. The median relative CTC_SC count and the median relative secretion of PSA, IL- 6 , and IL-8 during the settlement period were the most dynamic markers (Figure 2a,c,e). The CTC count continually changed from 95 to $300 \%$ from visit 2 to visit 4 . In contrast, the relative CTC_CC counts demonstrated a decreasing level during the period of analysis. The lowest relative CTC_CC count was reduced by $14 \%$ at visit 4 (Figure $2 \mathrm{~b}$ ). The median PSA level also showed variations with a range of $36 \%$ at visit 3 and $157 \%$ at visit 6 . The DKK-1 protein showed a relatively constant secretion of 90.1-112.5\%. IL-6 secretion remained relatively constant in the range of $126-117 \%$ until the fifth month. Interestingly, IL-6 secretion increased $440 \%$ in the 6th month. sHER-2 showed variations in a range of $96-$ $66.8 \%$, which revealed a continuous decrease in concentration under therapy. The serial change in IL-8 secretion demonstrated a variation of $66 \%$ in the third month to $156 \%$ in the 6th month. CD44 secretion was relatively constant over the observation period (104-86\%). 
Table 2. Serum levels of different biomarkers.

\begin{tabular}{ccccc}
\hline Median (Range) & mCRPC V1-V6 & PCa-1 & V2 + V3 & $p$-Value \\
\hline CD44 $(\mathrm{pg} / \mathrm{mL})$ & $710(205.9-4878)$ & $777.1(230.6-3382)$ & $783.6(386-2440)$ & 0.70 \\
\hline DKK-1 $(\mathrm{pg} / \mathrm{mL})$ & $4625(566.9-8878)$ & $3939(1632-10937)$ & $3976(1273-7988)$ & 0.80 \\
\hline sHer2 $(\mathrm{ng} / \mathrm{mL})$ & $3.3(0.83-16.46)$ & $3.3(1.1-7.7)$ & $3.5(1.27-8.4)$ & 0.39 \\
\hline IL-6 $(\mathrm{pg} / \mathrm{mL})$ & $11.7(1.91-180)$ & $5.6(1.5-587.2)$ & $8.2(1.0-589)$ & 0.24 \\
\hline IL-8 $(\mathrm{pg} / \mathrm{mL})$ & $20(1.98-112.7)$ & $10.8(4.8-1127)$ & $13.2(2.6-1216)$ & 0.27 \\
\hline CTC_CC & $4(0-820)$ & $0(0-5)$ & 0 & $<0.0001$ \\
\hline CTC_CS & $8.5(0-1428)$ & $0(0-1)$ & $0.04(0.04-1.12)$ & $<0.0001$ \\
\hline PSA $(\mathrm{ng} / \mathrm{mL})$ & $18.5(1-1120)$ & $8.2(0.64-38.8)$ & n.d. & 0.0001 \\
\hline CRP $(\mathrm{ng} / \mathrm{mL})$ & $7.3(1.8-94.8)$ & $2.1(1-26.4)$ & 0.03 \\
\hline
\end{tabular}

CD44-cluster of differentiation 44; DKK-1—Dickkopf1; sHER2—soluble human epidermal growth factor receptor 2; IL-6, -8-interleukin$6,-8$; CTC_CC—determined with CellCollector ${ }^{\circledR}$; CTC_CS—determined with the CellSearch ${ }^{\circledR}$ system; PSA-prostate-specific antigen; $\mathrm{CRP}-\mathrm{C}$-reactive protein.
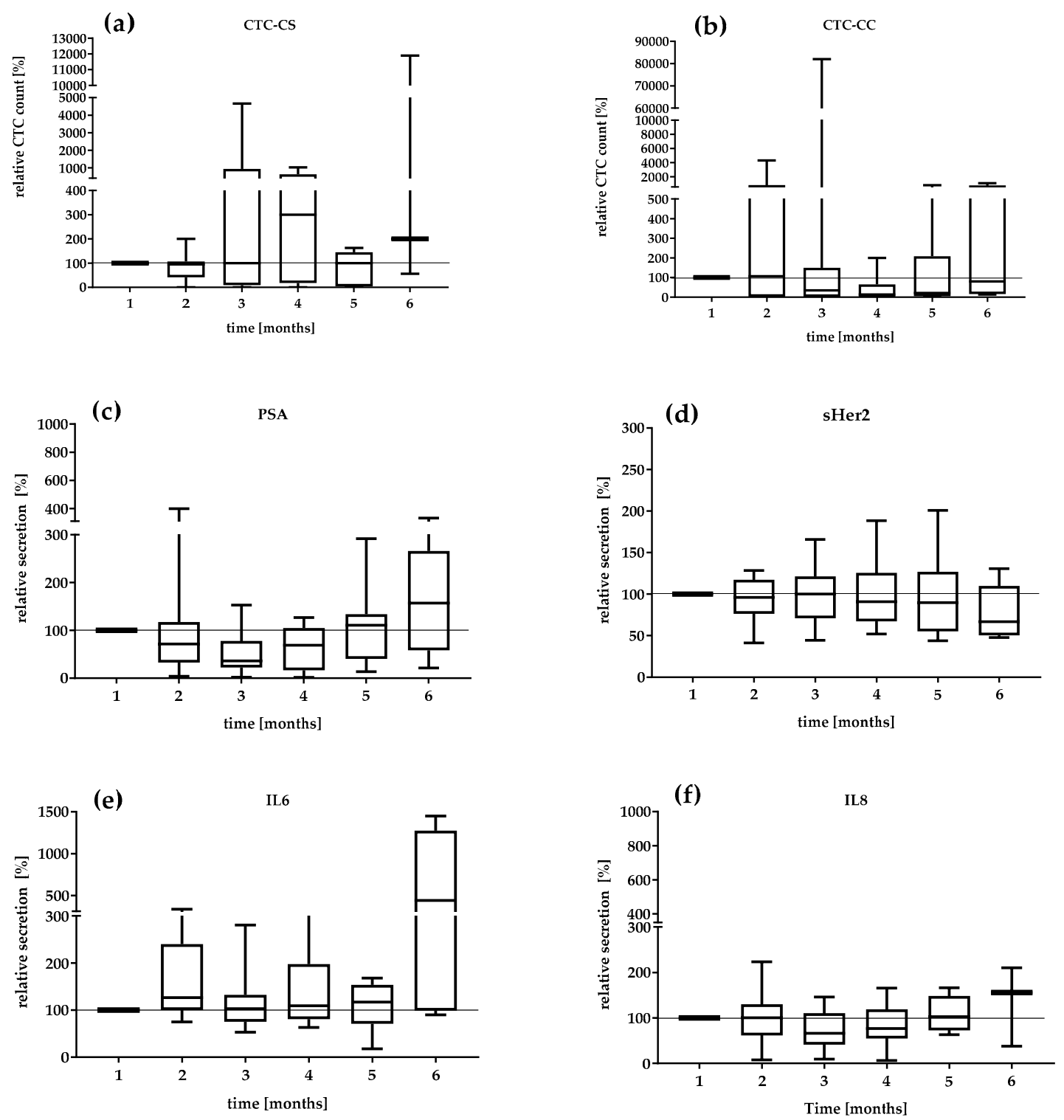

Figure 2. Cont. 

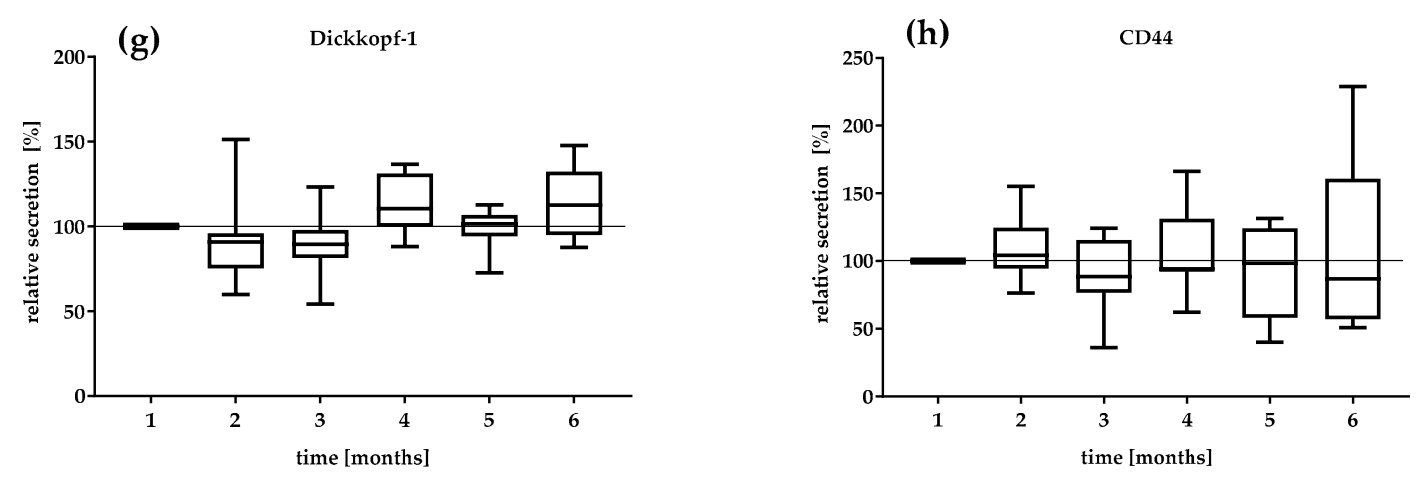

Figure 2. Relative value in percent of (a) CTC_CS count, (b) CTC_CC count, (c) PSA, (d) sHER2, (e) IL-6, (f) IL-8, (g) DKK-1, and (h) CD44 in the mCRPC patients during 6 months. The median relative secretion with minimum and maximum values. The value of the first visit was defined as $100 \%$.

The correlation of serial CTC secretion between the serial secretion of biomarkers and inflammatory markers is shown in Figure 3. CTC counts determined with the CellSearch ${ }^{\circledR}$ system (CTC_CS) were moderately positively correlated with the concentrations of DKK-1 $\left(\mathrm{r}_{\mathrm{S}}=0.35, p=0.01\right)$ and sHER-2 $\left(\mathrm{r}_{\mathrm{S}}=0.41, p=0.004\right)$ in the mCRPC patients. A strong correlation was found between the CTC_CS count and the PSA concentration $\left(\mathrm{r}_{\mathrm{s}}=0.75\right.$, $p \leq 0.0001)$ and the CTC counts of both platforms $\left(r_{s}=0.78, p=0.03\right)$. Within regard to the CTC count captured by CellCollector ${ }^{\circledR}$ (CTC_CC), no significant association was observed with the concentrations of the other blood-based biomarkers. The CRP concentration was strongly positively, but not significantly correlated with the CTC count (CTC_CS $\mathrm{r}_{\mathrm{S}}=0.60$, $\left.p=0.4 ; \mathrm{CTC}_{-} \mathrm{CC} \mathrm{r}_{\mathrm{s}}=0.78, p=0.078\right)$. Interestingly, we demonstrated a good correlation between PSA and sHer2 levels $\left(r_{s}=0.55, p \leq 0.0001\right)$ and PSA and IL-8 levels $\left(r_{s}=0.47\right.$, $p \leq 0.0001)$ in our cohort. For IL-6, a good negative correlation was observed with DKK-1 $\left(\mathrm{r}_{\mathrm{s}}=-0.45\right)$ and CD44 $\left(\mathrm{r}_{\mathrm{s}}=-0.54\right)$.

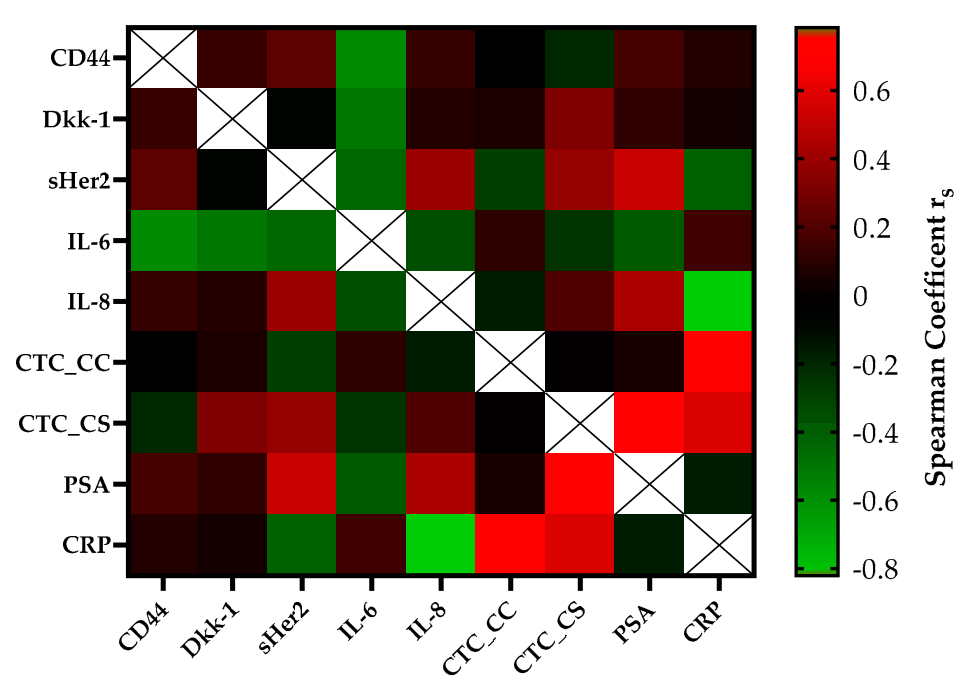

Figure 3. The heatmap of the correlation coefficients (Spearman) among biomarkers of the mCRPC patients. The color-coded correlation is on the left, where red demonstrates a strong positive correlation and light green indicates a strong negative correlation.

In the PCa-1 patient group, no significant correlation was found between the CTC counts of either platform and the biomarker levels; however, the levels of markers prior to prostate removal were correlated (Supplementary Figure S1).

The sensitivity, specificity, and area under the curve (AUC) value were determined for CTC_CC, DKK-1, PSA, CTC_CS, and sHER2, which were correlated significantly with the 
CTC_SC count. The results demonstrated that for a survival time of 24 months, the AUC values of these markers were $0.63,0.62,0.9,0.95$, and 0.79 , respectively. The PSA level and the CTC_CS showed the strongest ability to predict survival for 24 months for the mCRPC patients. These results were calculated with the 6-month median level of the evaluated markers for every single mCRPC patient (Figure 4).

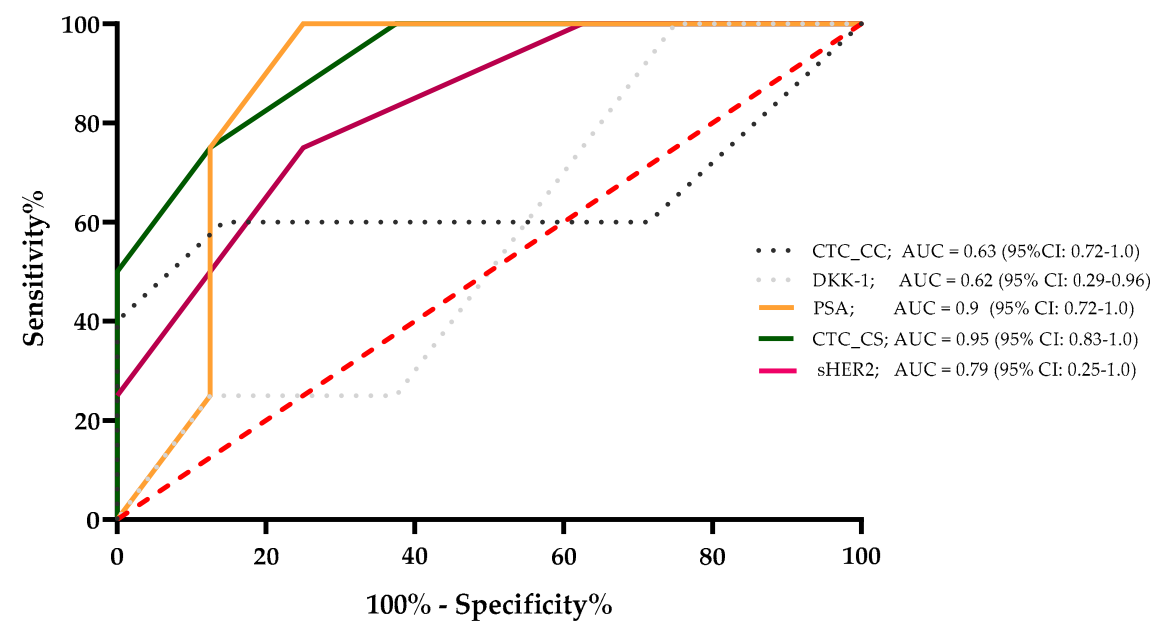

Figure 4. A survival ROC curve was plotted to evaluate the sensitivity, specificity, and AUC of serum concentrations of sHer2, PSA, DKK-1, CTC_CC, and CTC_CS and the 24-month survival.

\subsection{The OS Value of CTC Count Versus PSA Level}

We reached a follow-up time of 5 years in the study population, and compared the prognostic value of the median CTC count and the PSA concentration. In our analyses, we used the established CTC cutoff values of $<5$ or $\geq 5$ CTCs [32]. For the PSA level, we used the estimated cutoff value of $53 \mathrm{ng} / \mathrm{mL}$, which was calculated for the $\mathrm{mCRPC}$ patients in our study. In this study, the positive likelihood ratio was 8.0 (sensitivity $100 \%$, specificity 87.5) for PSA cutoff values of $53 \mathrm{ng} / \mathrm{mL}$.

Patients $(75 \%)$ with evaluated CTC counts of $<5$ cells survived 34 months, with a median of 56 months. Patients (75\%) with an evaluated CTC count of $\geq 5$ cells survived 14 months, with a median survival time of 21.5 months. The hazard ratio (HR), referring to $<5$ or $\geq 5$ CTCs, was 4.6 (95\% confidence interval (CI): 1.2-17.03; Figure 5a).

(a)

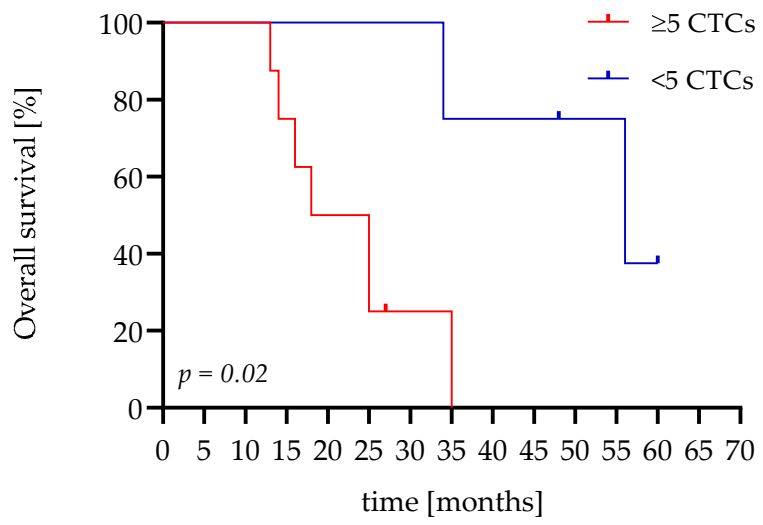

(b)

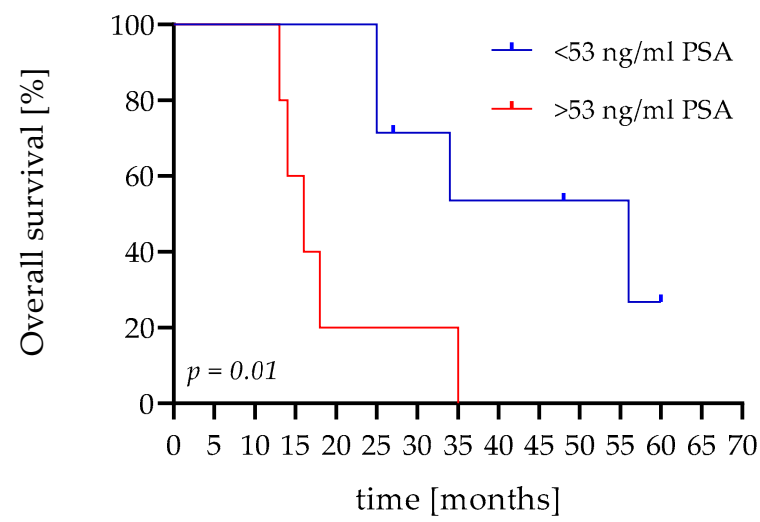

Figure 5. Comparison of Kaplan-Meier curves for OS according to the CTC count and PSA level of the mCRPC patients. (a) The patient shows $<5$ CTCs and a $\geq 5$ CTC difference in OS (56 months versus 21.5 months (HR 4.6, 95\% CI, 1.2-17)). (b) The patient shows a PSA level $<53 \mathrm{ng} / \mathrm{mL}$ and a $\geq 53 \mathrm{ng} / \mathrm{mL}$ difference in OS (56 months versus 16 months (HR 4.4 , 95\% CI, 0.9-21)). 
In comparison, with a PSA level $<53 \mathrm{ng} / \mathrm{mL}, 71.5 \%$ of the patients survived 25 months with a median of 56 months. Patients $(60 \%)$ with PSA levels $>53 \mathrm{ng} / \mathrm{mL}$ survived 14 months and had a median survival time of 16 months. The HR, referred to as $<53 \mathrm{ng} / \mathrm{mL}$ PSA or $>53 \mathrm{ng} / \mathrm{mL}$ PSA, was 4.4 (95\% CI: 0.9-21; Figure 5b).

\section{Discussion}

We analyzed the association between inflammatory markers and different biomarkers under therapy in a cohort of patients with PCa-l and mCRPC. Moreover, we compared two CTC isolation platforms for their sensitivity and specificity. There are many CTC isolation platforms; however, all of them have disadvantages and advantages [35,36]. We used CellCollector ${ }^{\circledR}$, an in vivo CTC isolation system [30,37], and the FDA-approved CellSearch ${ }^{\circledR}$ system [32]. Similarly, in both platforms, CTCs were captured using antibodies against the EpCAM protein and were further characterized. The CellSearch ${ }^{\circledR}$ system required a blood sample of $7.5 \mathrm{~mL}$, while CellCollector ${ }^{\circledR}$ required a larger volume. The CellSearch ${ }^{\circledR}$ system detected a higher CTC count in the mCRPC group, although the detection rate of CellCollector ${ }^{\circledR}$ was $84 \%$ compared with $73.5 \%$ of the CellSearch ${ }^{\circledR}$ system. Nevertheless, a range of 0-9 CTCs detected using CellCollector ${ }^{\circledR}$ in PCa-1 patients compared with a range of $0-1$ CTCs detected using the CellSearch ${ }^{\circledR}$ system. These results indicated that CellCollector ${ }^{\circledR}$ might be more useful than the CellSearch ${ }^{\circledR}$ system in nonmetastatic PCa patients because of the higher CTC detection rate. A possible reason for the different results could be the different EpCAM antibodies with differences in the affinity to the EpCam molecule. Furthermore, the veins in localized PCa patients are sometimes better for the in vivo application of the CellCollector ${ }^{\circledR}$ as in mCRPC patients. Even if the number of detected CTCs in indolent localized patients is low and their clinical utility remains unclear, their better specified molecular characterization would be crucial for clinical application. Chen et al. [38] further assessed high-risk nonmetastatic PCa patients and described CellCollector ${ }^{\circledR}$ as an efficient CTC technology for monitoring cancer relapse in localized PCa, as well as for monitoring of the treatment response.

The CTC counts obtained with CellCollector could also be tested in metastatic castration-sensitive prostate cancer patients (mCSPC) as biomarkers for evaluation of the treatment with androgen-receptor-axis-targeted (ARAT) therapy compared with docetaxel to improve the outcome in mCSPC patients $[39,40]$.

However, in a comparison of different CTC platforms (CellCollector ${ }^{\circledR}$, dual fluoroEPISPOTPSA/FGF2, and the CellSearch ${ }^{\circledR}$ system), the CellSearch ${ }^{\circledR}$ system was the most accurate predictor of metastatic PCa (AUC 0.76, 95\% CI: 0.631-0.908) [41]. Our ROC analysis showed an AUC of 0.95 (95\% CI: 0.83-1.0) for the CellSearch ${ }^{\circledR}$ system, which confirmed the high sensitivity and specificity of this system. The PSA level, a classic marker in blood-based therapeutic monitoring of advanced PCa patients, demonstrated a comparatively high sensitivity and specificity with an AUC of 0.90 (95\% CI: 0.72-1.0) in our mCRPC patient cohort (Figure 4). Interestingly, our results demonstrated a good correlation between the PSA level and the CTC count determined with the CellSearch ${ }^{\circledR}$ System. CTCs are prognostic parameters in mCRPC patients, but are usually independent of the PSA levels [32,42-44]. In our Kaplan-Meier OS analysis, a CTC count of $\geq 5$ cells and $>53 \mathrm{ng} / \mathrm{mL}$ PSA showed nearly identical HRs (CTC count HR $=4.6, p=0.02$ and PSA level HR $=4.4, p=0.01$ ). Our data showed that the CTC count and the PSA value in our cohort of mCRPC patients presented almost identical prognostic values. Nevertheless, CTCs can provide additional cancer-specific characteristics at the protein, mRNA, and DNA levels [35].

Furthermore, we found elevated serum levels of sHER2, DKK-1, IL-6, and IL-8 in the mCRPC patients and the PCa-l patients and found no significant difference between the groups (Table 2). Moreover, all of the analyzed markers were actively or passively involved in the bypassing of the AR signaling and might indicate active signaling in the blood. These factors may also influence the ability of CTCs to enhance inflammatory factors and biomarker release in blood circulation for possible crosstalk with cells. 
Moreover, we found that the median DKK-1 serum level of $4625 \mathrm{pg} / \mathrm{mL}$ in the mCRPC patients was slightly increased compared with that in the PCa-1 patients $(3939 \mathrm{pg} / \mathrm{mL})$, which may contribute to the development of osteoblastic metastasis. In addition, the higher DKK-1 concentration could indicate a possible switch in phenotype to the osteoblastic metastasis type [45]. In the serial measurements, a variation of $90.1-112.5 \%$ of DKK-1 was observed (Figure $3 \mathrm{~g}$ ). Interestingly, in the sixth month of systemic therapy, an increase of $112 \%$ was observed, as well as increases in the levels of PSA (157\%), IL-6 (440\%), CTC_CS $(200 \%)$, and IL-8 (156\%), which was consistent with the docetaxel treatment interruption (Figure 3). The doubling of the median CTC count suggests active cancer communication or micrometastatic progression. The increased serum level of DKK-1 could be due to the zoledronic acid treatment of the mCRPC patients, as shown by Thiele et al. [46] in an analysis of serum samples at different PCa stages. Our mCRPC cohort was under zoledronic acid treatment.

However, a good negative correlation of $-0.45(p<0.0001)$ was demonstrated for DKK1 and IL-6. This effect was described in inflamed joints of rheumatoid arthritis [47]. The median IL- 6 and IL- 8 concentrations in the serum of the MCRPC patients were substantially increased compared with those in the PCa-1 group (Table 2). Culig [48] postulated in his review that serum IL-6 can act as an attractant for tumor cells and is linked to aggressive tumors. The IL- 6 concentration of our cohort $(11.7 \mathrm{pg} / \mathrm{mL}$ in the $\mathrm{mCRPC}$ group) was similar to that of the cohort of Nakashima et al. [49], and higher than $7 \mathrm{pg} / \mathrm{mL}$. The increase of $440 \%$ in the sixth month of treatment could indicate active signaling pathways in PCa. Our results confirmed the findings from these studies, which concluded that higher IL-6 serum levels were correlated with the tumor stage and were inversely correlated with tumor survival and therapeutic response $[18,48]$. In the monitoring of mCRPC patients, the IL-8 level increased to $156 \%$ (visit at 6 months) compared with the baseline level (100\%). Maynard et al. [50] reported that the high expression of IL-8 in the tumor microenvironment is associated with aggressive PCa and with the loss of the AR. Analysis of the IL- 8 serum level of PCa-1 patients found no correlation with diagnosis and aggressiveness [51]. We also detected lower IL- 6 and IL- 8 concentrations in the serum of the PCa-1 group. In the mCRPC group, we could not demonstrate any significant correlation of interleukins 6 and 8 with the CTC count. One possible explanation could be the CTC status in the blood circulation and current tumor stage, which need to be explored in further studies. It is known that CTCs undergo a phenotype switch from epithelial to mesenchymal transition (EMT), and present a mesenchymal status [52]. Patients with newly diagnosed metastatic castrationsensitive PCa and positive for mesenchymal CTCs show a decline in resistance to androgen deprivation therapy compared with patients who are negative for EMT CTCs [53].

Interestingly the serum level of the sHER2 showed a significant $(p<0.001)$ moderate $\left(r_{\mathrm{s}}=0.41\right)$ correlation with the CTC_SC count. Although we could detect sHER2 in the serum, the median concentration was equal in our groups, but the range $(0.83-16.46 \mathrm{ng} / \mathrm{mL})$ in the mCRPC group was much wider. This finding suggests that CTCs in the blood circulation express HER2, and that HER2 signaling is activated through the cleavage of sHER2 (ECD). The single patient profile shows an increasing sHER2 concentration in the fifth and sixth months (data not shown), which is consistent with chemotherapy interruption. Josefsson et al. [54] demonstrated a high correlation between HER2 expression in CTCs and metastatic samples, and emphasized the potential for CTC phenotyping for individualized therapy in metastatic PCa. Furthermore, it was demonstrated in 236 PCa patients that HER2 over expression is associated with a low expression of the tumor suppressor gen PTEN (phosphatase and tensin homologue) and reduced the cancer-specific survival [55]. Using the AdnaTest ProstateCancerSelect/Detect kit for CTC isolation from the PCa patients in their study, they captured CTCs with the EpCAM and HER2 protein [54]. The same kit was used for the analysis by Antonarakis et al. [56]. This group detected AR splice variant 7 mRNA (AR-V7) in the CTCs from patients with castration-resistant PCa. The CTCs also express HER2 and AR-V7. This variant of the AR in CTCs has no ligand-binding domain, but via an active HER2 signaling it can bypass the androgen signaling pathway. In 
a recently published study, the expressions of AR-V7 and PTEN were determined in CTC. The authors demonstrated that more than two PTEN negative CTCs were associated with a 3.96 hazard ratio for progression or death compared with CRPC patients with less than two PTEN negative CTCs. Moreover, a high CTC AR-V7 positive count (0-20) was associated with a radiographic progression-free survival in ezalutamid-treated patients [57].

We determined the CD44 expression in the serum, but the median concentration in the mCRPC group was only slightly decreased compared with that in the PCa-l patients. However, the concentration range was much wider in this group than in the PCa-l group. Nevertheless, we could not observe any increase in the CD44 concentration after chemotherapy in the mCRPC group. Some patients demonstrated constant levels, and others had decreased levels after chemotherapy. Ma et al. [25] showed the interaction between CD44 and HER2 in PCa cell line, and linked this relationship to potential radio resistance PCa.

In this study, we showed that the CTC count determined with the CellSearch ${ }^{\circledR}$ system (CTC_CS) is more suitable for mCRPC patients than CellCollector, an in vivo isolation system. We identified a moderate correlation between the CTC counts and the biomarkers sHER2 and DKK-1, and a strong correlation with the PSA level. Additionally, we found that a CTC_CS count $\geq 5$ cells and a PSA level $>53 \mathrm{ng} / \mathrm{mL}$ presented approximately the same diagnostic potency with regard to the sensitivity and specificity for OS in our mCRPC patients. Furthermore, for better personalized characterization, it is crucial to expand the research focused on CTC phenotyping, and the interactions of these cells with coexisting, tumor-associated blood-released factors.

The limitations of our preliminary investigations are of course the small number of patients and the heterogeneous group of MCRPC patients (first and second line of chemotherapy). Likewise, the CTC platforms used here capture CTCs with an EpCam antibody but not CTCs with a mesenchymal phenotype. Moreover, we included no independent cohorts such as age match healthy woman or man. A wider characterization might provide additional information about the association between CTC and other biomarkers [13]. Lager studies are needed to further validate our findings.

Supplementary Materials: The following are available online at https: / www.mdpi.com/article / 10.3390/life11070664/s1, Figure S1: Heatmap of non-significant correlation coefficients (Spearman) among biomarkers of localized prostate cancer patients.

Author Contributions: conceptualization, G.T. and C.L.; methodology, G.T.; software, J.B.; validation, G.T., J.B. and P.F.; formal analysis, C.L.; investigation, G.T.; resources, P.F.; data curation, J.B.; writingoriginal draft preparation, G.T.; writing—review and editing, J.B.; visualization, C.L.; supervision, P.F.; project administration, G.T.; funding acquisition, G.T. All authors have read and agreed to the published version of the manuscript.

Funding: This research was funded by GILUPI GMPH. They provided CellCollector and the patients received travel expenses.

Institutional Review Board Statement: The study was conducted according to the guidelines of the Declaration of Helsinki and was approved by the medical faculty ethics committee of Martin Luther University Halle-Wittenberg (protocol code SMW EPCAM-Prostata-M00 and approved on 01/2011).

Informed Consent Statement: Informed consent was obtained from all of the subjects involved in the study.

Acknowledgments: We acknowledge the financial support within the funding program Open Access Publishing by the German Research Foundation (DFG).

Conflicts of Interest: The authors declare no conflict of interest. The funders had no role in the design of the study; in the collection, analyses, or interpretation of data; in the writing of the manuscript; or in the decision to publish the results. 


\section{References}

1. Bray, F.; Ferlay, J.; Soerjomataram, I.; Siegel, R.L.; Torre, L.A.; Jemal, A. Global cancer statistics 2018: GLOBOCAN estimates of incidence and mortality worldwide for 36 cancers in 185 countries. CA Cancer J. Clin. 2018, 68, 394-424. [CrossRef]

2. Bell, K.J.; Del Mar, C.; Wright, G.; Dickinson, J.; Glasziou, P. Prevalence of incidental prostate cancer: A systematic review of autopsy studies. Int. J. Cancer 2015, 137, 1749-1757. [CrossRef] [PubMed]

3. Jemal, A.; Culp, M.B.; Ma, J.; Islami, F.; Fedewa, S. Prostate Cancer Incidence 5 Years After US Preventive Services Task Force Recommendations Against Screening. J. Natl. Cancer Inst. 2021, 113, 64-71. [CrossRef] [PubMed]

4. Siegel, R.L.; Miller, K.D.; Jemal, A. Cancer statistics, 2019. CA Cancer J. Clin. 2019, 69, 7-34. [CrossRef]

5. Gandaglia, G.; Abdollah, F.; Schiffmann, J.; Trudeau, V.; Shariat, S.F.; Kim, S.P.; Perrotte, P.; Montorsi, F.; Briganti, A.; Trinh, Q.-D.; et al. Distribution of metastatic sites in patients with prostate cancer: A population-based analysis. Prostate 2014, 74, 210-216. [CrossRef]

6. Welch, D.R.; Hurst, D.R. Defining the Hallmarks of Metastasis. Cancer Res. 2019, 79, 3011-3027. [CrossRef]

7. Carceles-Cordon, M.; Kelly, W.K.; Gomella, L.; Knudsen, K.E.; Rodriguez-Bravo, V.; Domingo-Domenech, J. Cellular rewiring in lethal prostate cancer: The architect of drug resistance. Nat. Rev. Urol. 2020, 17, 292-307. [CrossRef] [PubMed]

8. Nuhn, P.; De Bono, J.S.; Fizazi, K.; Freedland, S.J.; Grilli, M.; Kantoff, P.W.; Sonpavde, G.; Sternberg, C.N.; Yegnasubramanian, S.; Antonarakis, E.S. Update on Systemic Prostate Cancer Therapies: Management of Metastatic Castration-resistant Prostate Cancer in the Era of Precision Oncology. Eur. Urol. 2019, 75, 88-99. [CrossRef]

9. Lorente, D.; Ravi, P.; Mehra, N.; Pezaro, C.; Omlin, A.; Gilman, A.; Miranda, M.; Rescigno, P.; Kolinsky, M.; Porta, N.; et al. Interrogating Metastatic Prostate Cancer Treatment Switch Decisions: A Multi-institutional Survey. Eur. Urol. Focus 2018, 4, 235-244. [CrossRef]

10. Caffo, O.; Maines, F.; Kinspergher, S.; Veccia, A.; Messina, C. Sequencing strategies in the new treatment landscape of prostate cancer. Futur. Oncol. 2019, 15, 2967-2982. [CrossRef]

11. Mottet, N.; van den Bergh, R.C.; Briers, E.; Van den Broeck, T.; Cumberbatch, M.G.; De Santis, M.; Cornford, P. EAU—EANMESTRO_ESUR_SIOG Guidelines on Prostate Cancer; European Association of Urology: Arnhem, The Netherlands, 2019.

12. Massague, J.; Obenauf, A.C. Metastatic colonization by circulating tumour cells. Nature 2016, 529, 298-306. [CrossRef]

13. Lambert, A.W.; Pattabiraman, D.R.; Weinberg, R.A. Emerging Biological Principles of Metastasis. Cell 2017, 168, 670-691. [CrossRef]

14. Lozar, T.; Gersak, K.; Cemazar, M.; Kuhar, C.G.; Jesenko, T. The biology and clinical potential of circulating tumor cells. Radiol. Oncol. 2019, 53, 131-147. [CrossRef] [PubMed]

15. Araki, S.; Omori, Y.; Lyn, D.; Singh, R.K.; Meinbach, D.M.; Sandman, Y.; Lokeshwar, V.B.; Lokeshwar, B.L. Interleukin-8 Is a Molecular Determinant of Androgen Independence and Progression in Prostate Cancer. Cancer Res. 2007, 67, 6854-6862. [CrossRef]

16. Waugh, D.J.; Wilson, C. The interleukin-8 pathway in cancer. Clin. Cancer Res. 2008, 14, 6735-6741. [CrossRef] [PubMed]

17. Aguilar-Saavedra, J.A.; Anjos, N.; Cantrill, R.; Carvalho, J.; Castro, N.F.; Conde Muiño, P. Search for magnetic monopoles in sqrt[s]=7 TeV pp collisions with the ATLAS detector. Phys. Rev. Lett. 2012, 109, 261803.

18. Nguyen, D.P.; Li, J.; Tewari, A.K. Inflammation and prostate cancer: The role of interleukin 6 (IL-6). BJU Int. 2014, 113, 986-992. [CrossRef] [PubMed]

19. Ueda, T.; Bruchovsky, N.; Sadar, M. Activation of the Androgen Receptor N-terminal Domain by Interleukin-6 via MAPK and STAT3 Signal Transduction Pathways. J. Biol. Chem. 2002, 277, 7076-7085. [CrossRef] [PubMed]

20. Culig, Z. Interleukin-6 Function and Targeting in Prostate Cancer. Adv. Exp. Med. Biol. 2021, 1290, 1-8. [CrossRef]

21. Qiu, Y.; Ravi, L.; Kung, H.-J. Requirement of ErbB2 for signalling by interleukin-6 in prostate carcinoma cells. Nat. Cell Biol. 1998, 393, 83-85. [CrossRef]

22. Perrier, A.; Gligorov, J.; Lefevre, G.; Boissan, M. The extracellular domain of Her2 in serum as a biomarker of breast cancer. Lab. Investig. 2018, 98, 696-707. [CrossRef]

23. Craft, N.; Shostak, Y.; Carey, M.; Sawyers, C.L. A mechanism for hormone-independent prostate cancer through modulation of androgen receptor signaling by the HER-2/neu tyrosine kinase. Nat. Med. 1999, 5, 280-285. [CrossRef]

24. Miller, D.R.; Ingersoll, M.A.; Lin, M.-F. ErbB-2 signaling in advanced prostate cancer progression and potential therapy. EndocrineRelat. Cancer 2019, 26, R195-R209. [CrossRef]

25. Ma, J.-W.; Wang, X.; Chang, L.; Zhong, X.-Y.; Jing, H.; Zhu, X.; Wang, S.; Xiao, W. CD44 collaborates with ERBB2 mediate radiation resistance via p38 phosphorylation and DNA homologous recombination pathway in prostate cancer. Exp. Cell Res. 2018, 370, 58-67. [CrossRef]

26. Hiraga, T.; Ito, S.; Nakamura, H. Cancer Stem-like Cell Marker CD44 Promotes Bone Metastases by Enhancing Tumorigenicity, Cell Motility, and Hyaluronan Production. Cancer Res. 2013, 73, 4112-4122. [CrossRef] [PubMed]

27. Senbanjo, L.T.; AlJohani, H.; Majumdar, S.; Chellaiah, M.A. Characterization of CD44 intracellular domain interaction with RUNX2 in PC3 human prostate cancer cells. Cell Commun. Signal. 2019, 17, 1-13. [CrossRef] [PubMed]

28. Baron, R.; Rawadi, G. Targeting the Wnt/beta-catenin pathway to regulate bone formation in the adult skeleton. Endocrinology 2007, 148, 2635-2643. [CrossRef] [PubMed]

29. Pakula, H.; Xiang, D.; Li, Z. A Tale of Two Signals: AR and WNT in Development and Tumorigenesis of Prostate and Mammary Gland. Cancers 2017, 9, 14. [CrossRef] [PubMed] 
30. Theil, G.; Boehm, C.; Fischer, K.; Bialek, J.; Hoda, R.; Weber, E.; Schönburg, S.; Kawan, F.; Fornara, P. In vivo isolation of circulating tumor cells in patients with different stages of prostate cancer. Oncol. Lett. 2021, 21, 1-8. [CrossRef]

31. Kuske, A.; Gorges, T.M.; Tennstedt, P.; Tiebel, A.-K.; Pompe, R.S.; Preißer, F.; Prues, S.; Mazel, M.; Markou, A.; Lianidou, E.; et al. Improved detection of circulating tumor cells in non-metastatic high-risk prostate cancer patients. Sci. Rep. 2016, 6, 39736. [CrossRef]

32. De Bono, J.S.; Scher, H.I.; Montgomery, R.B.; Parker, C.; Miller, M.C.; Tissing, H.; Doyle, G.V.; Terstappen, L.; Pienta, K.; Raghavan, D. Circulating Tumor Cells Predict Survival Benefit from Treatment in Metastatic Castration-Resistant Prostate Cancer. Clin. Cancer Res. 2008, 14, 6302-6309. [CrossRef]

33. Allard, W.J.; Matera, J.; Miller, M.C.; Repollet, M.; Connelly, M.C.; Rao, C.; Tibbe, A.G.J.; Uhr, J.W.; Terstappen, L.W.M.M. Tumor cells circulate in the peripheral blood of all major carcinomas but not in healthy subjects or patients with nonmalignant diseases. Clin. Cancer Res. 2004, 10, 6897-6904. [CrossRef] [PubMed]

34. Llopis, P.M.; Senft, R.A.; Ross-Elliott, T.J.; Stephansky, R.; Keeley, D.P.; Koshar, P.; Marqués, G.; Gao, Y.-S.; Carlson, B.R.; Pengo, T.; et al. Best practices and tools for reporting reproducible fluorescence microscopy methods. Nat. Methods 2021, 1-14. [CrossRef]

35. Theil, G.; Fornara, P.; Bialek, J. Position of Circulating Tumor Cells in the Clinical Routine in Prostate Cancer and Breast Cancer Patients. Cancers 2020, 12, 3782. [CrossRef]

36. Alix-Panabières, C.; Pantel, K. Technologies for detection of circulating tumor cells: Facts and vision. Lab Chip 2013, 14, 57-62. [CrossRef] [PubMed]

37. Saucedo-Zeni, N.; Mewes, S.; Niestroj, R.; Gasiorowski, L.; Murawa, D.; Nowaczyk, P.; Lücke, K. A novel method for the in vivo isolation of circulating tumor cells from peripheral blood of cancer patients using a functionalized and structured medical wire. Int. J. Oncol. 2012, 41, 1241-1250.

38. Chen, S.; Tauber, G.; Langsenlehner, T.; Schmölzer, L.M.; Pötscher, M.; Riethdorf, S.; Kuske, A.; Leitinger, G.; Kashofer, K.; Czyż, Z.T.; et al. In Vivo Detection of Circulating Tumor Cells in High-Risk Non-Metastatic Prostate Cancer Patients Undergoing Radiotherapy. Cancers 2019, 11, 933. [CrossRef]

39. Buonerba, C.; Ferro, M.; Dolce, P.; Crocetto, F.; Verde, A.; Lucarelli, G.; Scafuri, L.; Facchini, S.; Vaia, A.; Marinelli, A.; et al. Predictors of efficacy of androgen-receptor-axis-targeted therapies in patients with metastatic castration-sensitive prostate cancer: A systematic review and meta-analysis. Crit. Rev. Oncol. 2020, 151, 102992. [CrossRef]

40. Ferro, M.; Lucarelli, G.; Crocetto, F.; Dolce, P.; Verde, A.; La Civita, E.; Zappavigna, S.; de Cobelli, O.; Di Lorenzo, G.; Facchini, B.A.; et al. First-line systemic therapy for metastatic castration-sensitive prostate cancer: An updated systematic review with novel findings. Crit. Rev. Oncol. 2021, 157, 103198. [CrossRef]

41. Cieślikowski, W.A.; Budna-Tukan, J.; Świerczewska, M.; Ida, A.; Hrab, M.; Jankowiak, A.; Mazel, M.; Nowicki, M.; Milecki, P.; Pantel, K.; et al. Circulating Tumor Cells as a Marker of Disseminated Disease in Patients with Newly Diagnosed High-Risk Prostate Cancer. Cancers 2020, 12, 160. [CrossRef]

42. Lorente, D.; Olmos, D.; Mateo, J.; Dolling, D.; Bianchini, D.; Seed, G.; Flohr, P.; Crespo, M.; Figueiredo, I.; Miranda, S.; et al. Circulating tumour cell increase as a biomarker of disease progression in metastatic castration-resistant prostate cancer patients with low baseline CTC counts. Ann. Oncol. 2018, 29, 1554-1560. [CrossRef] [PubMed]

43. Scher, H.I.; Lu, D.; Schreiber, N.A.; Louw, J.; Graf, R.P.; Vargas, H.A.; Johnson, A.; Jendrisak, A.; Bambury, R.; Danila, D.; et al. Association of AR-V7 on Circulating Tumor Cells as a Treatment-Specific Biomarker with Outcomes and Survival in Castration-Resistant Prostate Cancer. JAMA Oncol. 2016, 2, 1441-1449. [CrossRef] [PubMed]

44. Scher, H.I.; Heller, G.; Molina, A.; Attard, G.; Danila, D.C.; Jia, X.; de Bono, J.S. Circulating tumor cell biomarker panel as an individual-level surrogate for survival in metastatic castration-resistant prostate cancer. J. Clin. Oncol. 2015, 33, 1348-1355. [CrossRef]

45. Aufderklamm, S.; Hennenlotter, J.; Leidenberger, P.; Rausch, S.; Hohneder, A.; Kühs, U.; Todenhöfer, T. Systemic Alterations of Wnt Inhibitors in Patients with Prostate Cancer and Bone Metastases. Dis. Markers 2018, 2018, 1874598. [CrossRef]

46. Thiele, S.; Rauner, M.; Goettsch, C.; Rachner, T.D.; Benad, P.; Fuessel, S.; Erdmann, K.; Hamann, C.; Baretton, G.B.; Wirth, M.P.; et al. Expression profile of WNT molecules in prostate cancer and its regulation by aminobisphosphonates. J. Cell. Biochem. 2011, 112, 1593-1600. [CrossRef]

47. Yeremenko, N.; Zwerina, K.; Rigter, G.; Pots, D.; Fonseca, J.E.; Zwerina, J.; Schett, G.; Baeten, D. Brief Report: Tumor Necrosis Factor and Interleukin-6 Differentially Regulate Dkk-1 in the Inflamed Arthritic Joint. Arthritis Rheumatol. 2015, 67, $2071-2075$. [CrossRef] [PubMed]

48. Culig, Z. Proinflammatory cytokine interleukin-6 in prostate carcinogenesis. Am. J. Clin. Exp. Urol. 2014, 2, 231-238. [PubMed]

49. Nakashima, J.; Tachibana, M.; Horiguchi, Y.; Oya, M.; Ohigashi, T.; Asakura, H.; Murai, M. Serum interleukin 6 as a prognostic factor in patients with prostate cancer. Clin. Cancer Res. 2000, 6, 2702-2706. [PubMed]

50. Maynard, J.P.; Ertunc, O.; Kulac, I.; Del Valle, J.A.B.; De Marzo, A.M.; Sfanos, K.S. IL8 Expression Is Associated with Prostate Cancer Aggressiveness and Androgen Receptor Loss in Primary and Metastatic Prostate Cancer. Mol. Cancer Res. 2019, 18, 153-165. [CrossRef]

51. Roumeguere, T.; Legrand, F.; El Rassy, E.; Kaitouni, M.I.; Albisinni, S.; Rousseau, A.; Vanhaeverbeek, M.; Rorive, S.; Decaestecker, C.; Debeir, O.; et al. A prospective clinical study of the implications of IL-8 in the diagnosis, aggressiveness and prognosis of prostate cancer. Futur. Sci. OA 2018, 4, FSO266. [CrossRef]

52. Armstrong, A.J. Epithelial-mesenchymal transition in cancer progression. Clin. Adv. Hematol. Oncol. $2011,9,941-943$. 
53. Yang, Y.-J.; Kong, Y.-Y.; Li, G.-X.; Wang, Y.; Ye, D.-W.; Dai, B. Phenotypes of circulating tumour cells predict time to castration resistance in metastatic castration-sensitive prostate cancer. BJU Int. 2019, 124, 258-267. [CrossRef] [PubMed]

54. Josefsson, A.; Larsson, K.; Månsson, M.; Bjorkman, J.; Rohlová, E.; Åhs, D.; Brisby, H.; Damber, J.-E.; Welén, K. Circulating tumor cells mirror bone metastatic phenotype in prostate cancer. Oncotarget 2018, 9, 29403-29413. [CrossRef]

55. Ahmad, I.; Gao, M.; Patel, R.; Leung, H.Y. Modelling synergistic interactions between HER2, Sprouty2 and PTEN in driving prostate carcinogenesis. Asian J. Androl. 2013, 15, 323-327. [CrossRef] [PubMed]

56. Antonarakis, E.S.; Lu, C.; Wang, H.; Luber, B.; Nakazawa, M.; Roeser, J.C.; Chen, Y.; Mohammad, T.A.; Chen, Y.; Fedor, H.L.; et al. AR-V7 and Resistance to Enzalutamide and Abiraterone in Prostate Cancer. N. Engl. J. Med. 2014, 371, 1028-1038. [CrossRef] [PubMed]

57. Di Lorenzo, G.; Zappavigna, S.; Crocetto, F.; Giuliano, M.; Ribera, D.; Morra, R.; Buonerba, C. Assessment of Total, PTEN(-), and AR-V7(+) Circulating Tumor Cell Count by Flow Cytometry in Patients with Metastatic Castration-Resistant Prostate Cancer Receiving Enzalutamide. Clin. Genitourin. Cancer 2021, in press. 